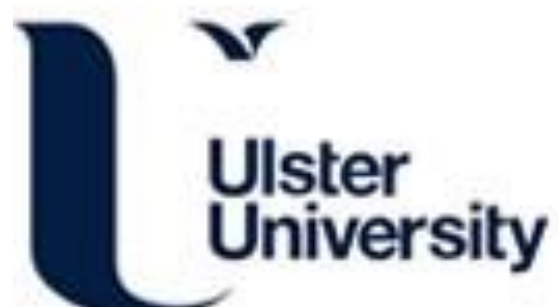

\section{Gas temperature and electron temperature measurements by emission spectroscopy for an atmospheric microplasma}

Mariotti, D., Shimizu, Y., Sasaki, T., \& Koshizaki, N. (2007). Gas temperature and electron temperature measurements by emission spectroscopy for an atmospheric microplasma. Journal of Applied Physics, 101(1), 013307-1. https://doi.org/10.1063/1.2409318

Link to publication record in Ulster University Research Portal

Published in:

Journal of Applied Physics

Publication Status:

Published (in print/issue): 01/01/2007

DOI:

10.1063/1.2409318

\section{Document Version}

Publisher's PDF, also known as Version of record

\section{General rights}

Copyright for the publications made accessible via Ulster University's Research Portal is retained by the author(s) and / or other copyright owners and it is a condition of accessing these publications that users recognise and abide by the legal requirements associated with these rights.

\section{Take down policy}

The Research Portal is Ulster University's institutional repository that provides access to Ulster's research outputs. Every effort has been made to ensure that content in the Research Portal does not infringe any person's rights, or applicable UK laws. If you discover content in the Research Portal that you believe breaches copyright or violates any law, please contact pure-support@ulster.ac.uk. 


\title{
Gas temperature and electron temperature measurements by emission spectroscopy for an atmospheric microplasma
}

\author{
Davide Mariotti, ${ }^{\text {a) }}$ Yoshiki Shimizu, Takeshi Sasaki, and Naoto Koshizaki \\ Nanoarchtectonics Research Center (NARC), National Institute of Advanced Industrial Science \\ and Technology (AIST), AIST Tsukuba Central 5, 1-1-1 Higashi, Tsukuba, Ibaraki 305-8565, Japan
}

(Received 18 September 2006; accepted 31 October 2006; published online 12 January 2007)

\begin{abstract}
A microplasma suitable for material processing at atmospheric pressure in argon and argon-oxygen mixtures is being studied here. The microplasma is ignited by a high voltage dc pulse and sustained by low power $(1-5 \mathrm{~W})$ at $450 \mathrm{MHz}$. the mechanisms responsible for sustaining the microplasma require a more detailed analysis, which will be the subject of further study. Here it is shown that the microplasma is in nonequilibrium and appears to be in glow mode. The effect of power and oxygen content is also analyzed in terms of gas temperature and electron temperature. Both the gas temperature and the electron temperature have been determined by spectral emission and for the latter a very simple method has been used based on a collisional-radiative model. It is observed that power coupling is affected by a combination of factors and that prediction and control of the energy flow are not always straightforward even for simple argon plasmas. Varying gas content concentration has shown that oxygen creates a preferential energy channel towards increasing the gas temperature. Overall the results have shown that combined multiple diagnostics are necessary to understand plasma characteristics and that spectral emission can represent a valuable tool for tailoring microplasma to specific processing requirements. (C) 2007 American Institute of Physics. [DOI: $10.1063 / 1.2409318]$
\end{abstract}

\section{INTRODUCTION}

A general trend in miniaturization has characterized many of the past decades of scientific progress and, for instance, in material science a nanoscale environment is routinely studied. In plasma science the miniaturized version of the widely used technological plasmas has interested scientists for some time and has found steadily application in plasma display technology for many years. ${ }^{1,2}$ Nevertheless only in the last decade researchers have increasingly studied microscale plasma in a wider sense, probably due to a growing belief in the potential applications. ${ }^{3,4}$ Microplasmas are now being considered in many and different fields including material processing, ${ }^{5-15}$ gas treatment, ${ }^{16}$ light sources, ${ }^{17}$ propulsion, ${ }^{18}$ analytical chemistry, ${ }^{19-24}$ photonics, ${ }^{25,26}$ and medical applications. ${ }^{27,28}$ Moreover the basic scientific interest is growing stronger as microplasma characteristics are unfolded and recognized to be different from the larger parents. ${ }^{26,29}$ In this scenario a noninvasive diagnostic technique such as emission spectroscopy becomes a very powerful tool. ${ }^{30}$

In this contribution a particular plasma configuration suitable for processing of nanostructured materials is reported and optical emission spectroscopy is used to evaluate the gas temperature and the electron temperature. Both the gas temperature and the electron temperature play a fundamental role in the evaluation of the microplasma characteristics. Diagnostic techniques for microplasmas are still limited and, in particular, for the electron temperature a simple method based on optical emission can be extremely practical considering the difficulties presented by other techniques

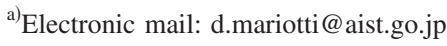

(e.g., Langmuir probe and Thomson scattering). The plasma is formed from a mixture of argon and oxygen in a microdiameter capillary. Argon-oxygen plasmas are widely used in material processing and the effect of oxygen on the plasma parameters is still an intriguing topic of research. ${ }^{31,32}$ The system configuration used here is partly new and utilizes power at $450 \mathrm{MHz}$ in atmospheric pressure. The plasma produced with this configuration is at least $1 \mathrm{~mm}$ long with a cross section of less than $0.2 \mathrm{~mm}$ in diameter and for this reason it is classified as a microplasma. The investigation overall aims to better understand the effect of power, gas flow, and oxygen relative content so that this microplasma technique could be better exploited for material processing of nanostructures.

\section{EXPERIMENTAL SETUP AND MICROPLASMA APPEARANCE}

\section{A. Description of the experimental arrangement}

The full experimental setup can be seen in Fig. 1. An ultrahigh frequency (UHF, $450 \mathrm{MHz}$ ) power supply is connected through a matching network to a small copper tube, about $7 \mathrm{~mm}$ long and with a $2 \mathrm{~mm}$ external diameter (0.15 mm thickness). The copper tube is then placed around a quartz glass capillary of $0.7 \mathrm{~mm}$ internal diameter $(0.15 \mathrm{~mm}$ thickness) where the gas mixture is injected at a variable flow rate. Inside the quartz capillary, a metal wire is also present which is inserted down to $1 \mathrm{~mm}$ distance from the copper cylinder. The visible plasma column is therefore $1 \mathrm{~mm}$ long. The wire used for this study is a titanium $0.3 \mathrm{~mm}$ diameter wire, which is also connected to a dc high voltage (HV) power supply. 


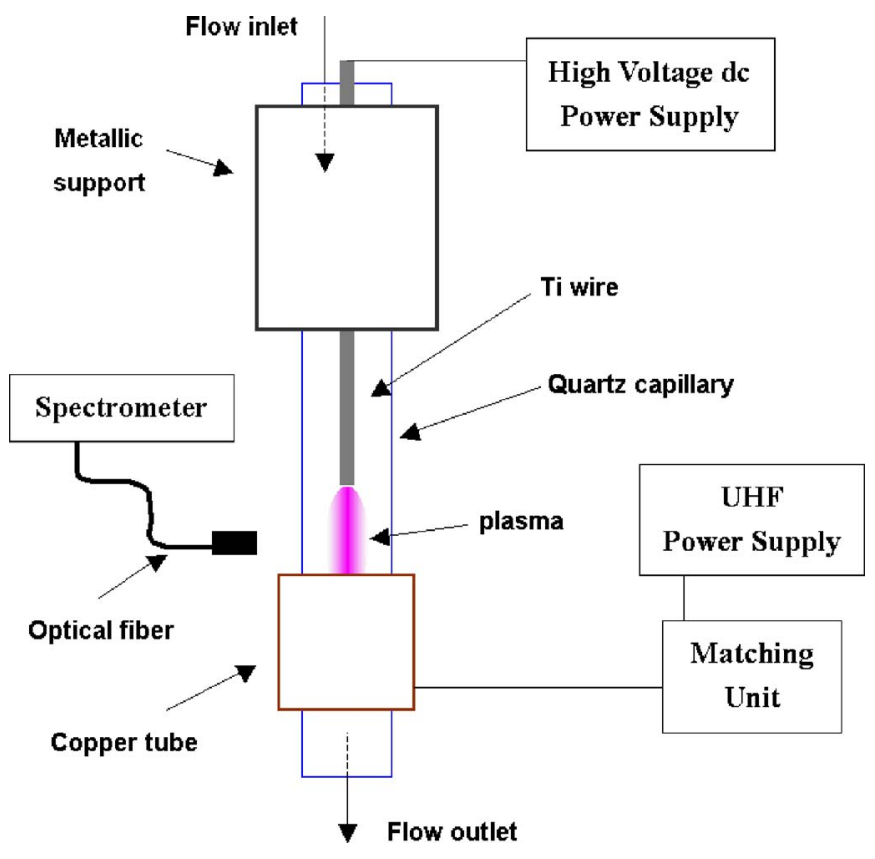

FIG. 1. (Color online) Schematic diagram of the microplasma system; in this drawing the relative dimensions do not represent the real system.

Plasma emission is collected by an optical fiber at about $3 \mathrm{~cm}$ distance from the microplasma. The fiber is connected to a wavelength calibrated HR2000 Ocean Optic spectrometer. The spectrometer has been calibrated with a certified tungsten lamp to produce corrected relative intensities. The spectrometer spectral broadening has been measured with a helium-cadmium laser and resulted to be about $1 \mathrm{~nm}$ full width at $1 / e$ of the maximum. Exposure time for the emission spectra was $100 \mathrm{~ms}$ and the average value over ten consecutive spectra was taken (wavelength range from about 250 to $1000 \mathrm{~nm}$ ). In order for the temperature measurements to be valid, the properties to be analyzed have to be assumed constant in the plasma volume where the emission originates and within the acquisition time frame. This assumption is probably justified for the gas temperature, while regarding the validity for the electron temperature measurements a more detailed discussion is given in one of the following sections.

\section{B. Experimental procedure}

For all the results presented here, the plasma is generated in the following steps. First the gas bottle is opened and the flow is run for several minutes at the desired settings $[10,15$, or 20 SCCM (SCCM denotes cubic centimeter per minute at STP)]. Premixed gas bottles supply the gas mixture of argon and $\mathrm{O}_{2}\left(0 \%, 0.02 \%, 0.2 \%\right.$, or $0.5 \%$ of $\left.\mathrm{O}_{2}\right)$. The power from the $450 \mathrm{MHz}$ power supply is then set to $20 \mathrm{~W}$. A pulse of about $5 \mathrm{kV}$ is applied to the $\mathrm{Ti}$ wire, which ignites the plasma and immediately the UHF power is reduced to the desired power setting to sustain the plasma $(1,3$, or $5 \mathrm{~W})$.

\section{Possible ignition mechanism and plasma appearance}

The microplasma is therefore ignited via dc discharge from the titanium wire within the capillary and then is sus-

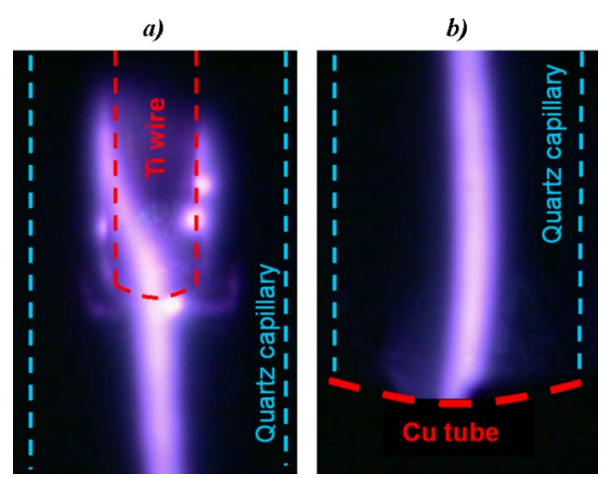

FIG. 2. (Color online) Microplasma column. (a) Top part near the titanium wire and (b) bottom part near the copper tube. The microplasma was sustained by $1 \mathrm{~W}$ UHF power with 20 SCCM flowing argon.

tained by the UHF power coupled through the copper tube. The high voltage discharge is believed to generate initial free electrons and probably contribute to an initial heating of all the system components. The titanium wire is likely to behave as an electron emitter under the combined effect of the electromagnetic field generated by the copper tube, by the bombardment of ions/energized atoms, and partly enhanced by an overall increased temperature.

After ignition and after the UHF power is reduced to the desired output, the microplasma stabilizes immediately. The power required to sustain the microplasma is very low, below $1 \mathrm{~W}$, while at power above $10 \mathrm{~W}$ the temperature tends to increase drastically leading to instabilities, capillary damage, and extinction. It has to be noticed that the titanium wire is not grounded but remains connected to the $\mathrm{HV}$ dc power supply even after the high voltage pulse ignition. Complete disconnection of the wire leaving it floating did not cause any noticeable change to the plasma state, observed by the naked eye.

The appearance of the microplasma is a diffuse luminous glow column (visible for $1 \mathrm{~mm}$ in length) that expands from the tip of the titanium wire and disappears in the copper cylinder within the capillary (Fig. 2). Varying the control parameters (UHF power, oxygen content, and flow rate), the diameter of the plasma column varies between 0.13 to $0.2 \mathrm{~mm}$; also the characteristic color is different. At certain settings of power, flow, and oxygen content outside of the ranges studied here, the microplasma column exhibits self-organized patterns similar to striations (Fig. 3). a)

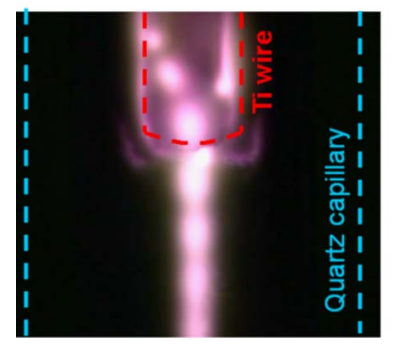

b)

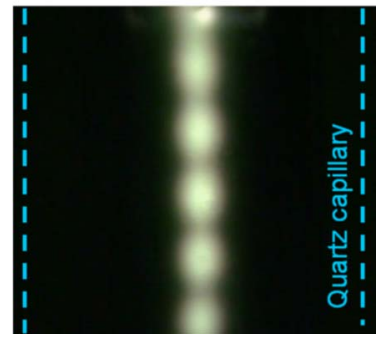

FIG. 3. (Color online) Self-organized patterns observed in the microplasma. (a) Top part near the titanium wire at $5 \mathrm{~W}$ and 6 SCCM flowing argon and (b) middle part at less than $1 \mathrm{~W}$ and 10 SCCM flowing argon. 


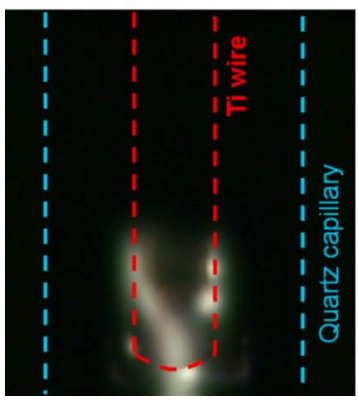

b)

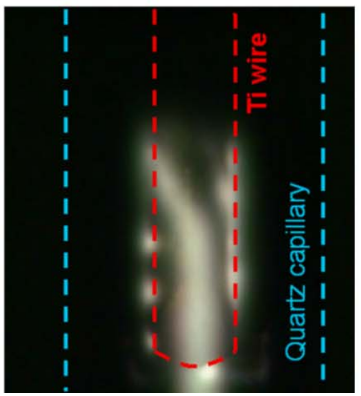

c)

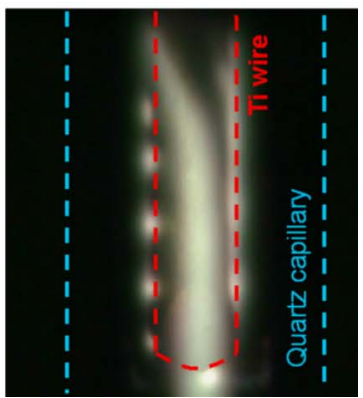

FIG. 4. (Color online) Images showing extension of the plasma to the surface of the titanium wire. The extension was generally observed for decreasing oxygen content and increasing power, from about 1 to about $5 \mathrm{~W}$ in the specific case of the images shown.
Depending on the settings, the microplasma may envelope the titanium wire with a very reproducible pattern (Fig. 4). In general the wire surface area that is surrounded by the plasma increases with increasing power, and decreases with increasing $\mathrm{O}_{2}$ content but does not vary with flow rate.

\section{METHODS USED TO DETERMINE GAS TEMPERATURE AND ELECTRON TEMPERATURE}

\section{A. Gas temperature}

As already mentioned the gas mixture used is argon with different concentrations of oxygen; nevertheless the emission from the second positive system of nitrogen is observed between 330 and $400 \mathrm{~nm}$. This is a common result observed in discharges with nitrogen impurities (Fig. 5). The presence of the $\mathrm{OH}$ emission band $\left(A^{2} \Sigma, \nu=0 \rightarrow X^{2} \Pi, \nu^{\prime}=0\right)$ between 306 and $309 \mathrm{~nm}$ is also quite frequent and is likely to originate from water vapor in the feed channels and in the capillary. ${ }^{17}$ This $\mathrm{OH}$ emission band has been very consistent in all experiments carried out (Fig. 5).

Both emissions from $\mathrm{N}_{2}$ and $\mathrm{OH}$ are suitable for gas temperature measurements. ${ }^{17,30,33-39}$ Unfortunately, in our measurements, nitrogen emission is often weak and seems to be affected by some other emission line; we suspect titanium emission lines which are present in the same spectral range and that might originate from etching of titanium atoms from the wire surface. ${ }^{40-42}$ Therefore, the $\mathrm{OH}$ band has been chosen for gas temperature measurements.

Comparing synthetic spectra with experimental spectra the gas temperature could be computed by minimizing the error between the two spectra. The method has been de-

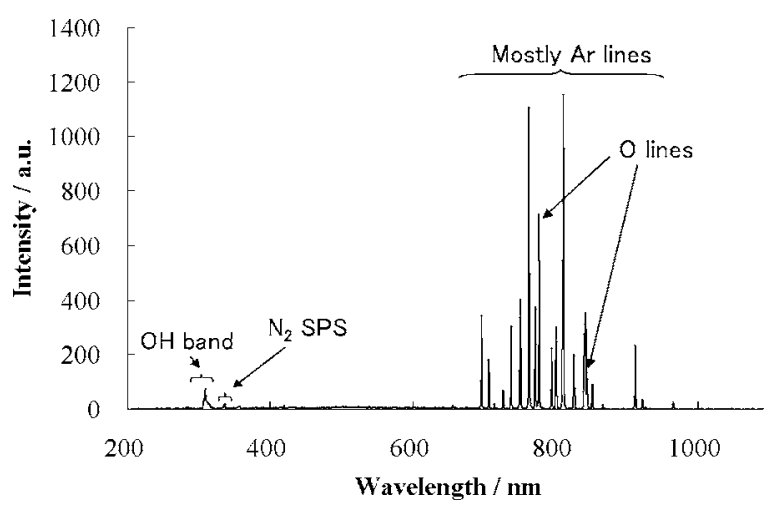

FIG. 5. Typical spectrum recorded. In this case the plasma was sustained by $5 \mathrm{~W}$ UHF power in a mixture of argon and $0.5 \% \mathrm{O}_{2}$ flowing at $20 \mathrm{SCCM}$ (SPS is second positive system). scribed in detail and validated elsewhere ${ }^{36,38}$ and relies on the assumption that rotational relaxation is much faster than vibrational and electronic transitions. The results are quite sensitive to the instrumental broadening parameter used for the computation of the synthetic spectra. In addition to the helium-cadmium laser, spectral broadening has been measured from atomic argon lines, providing a broadening value that depends on the wavelength. As a result, within the spectral range of the $\mathrm{OH}$ band, a linewidth from 1.025 to $1.019 \mathrm{~nm}$ at $1 / e$ of the maximum has been used. To further confirm our results we have also calculated gas temperature with different values for the linewidths. Some small variations in the absolute values of the temperature could be noticed; nevertheless the general trends have been confirmed in all cases.

\section{B. Electron temperature}

An interpretation of temperature in a strict thermodynamic sense is not possible for nonequilibrium plasmas; therefore temperatures have to be defined for different groups of particles. At the same time it is often the case that for electrons, which are of utmost importance in plasmas and discharges, the energy distribution does not follow a Maxwellian behavior in which case the temperature parameter has to be considered carefully.

There are three different trends in the interpretation of the electron temperature and generally accepted in the literature: (1) when the energy distribution is known to be Maxwellian we have a temperature in real Maxwellian sense, i.e., a Maxwellian electron temperature; (2) if no assumptions can be made on the particular electron distribution but an average electron energy is available then the electron temperature can be related to an equivalent Maxwellian distribution by the relation $T_{e}=(2 / 3)\langle\varepsilon\rangle$ (where $\langle\varepsilon\rangle$ is the average electron energy and $T_{e}$ is the electron temperature), ${ }^{43}$ in which case we would obtain an equivalent electron temperature; (3) finally, if other experimental evidence is available (such as spectral emission), which allows to model the results with a Maxwellian distribution, ${ }^{44-47}$ we would obtain an effective electron temperature.

The importance of the electron energy in material processing originates from the necessity to evaluate and predict the ability of nonequilibrium plasmas (and of the electrons) to produce radicals and promote specific reactions. An accurate measurement of the complete electron energy distribution function (EEDF) is certainly useful; nevertheless mea- 
surement techniques to obtain the EEDF can be difficult to implement. The use of the electron temperature parameters as described above is therefore extremely advantageous and practical to assess relative behavior of a plasma system.

In this work we do not have supportive experimental evidence to state that electrons assume a Maxwellian distribution and microplasmas can present rather peculiar characteristics. ${ }^{26,29}$ In lower pressure microplasmas, typical deviations from a Maxwellian distribution are observed in the form of a high-energy tail or an energetic electron beam. ${ }^{48}$ These deviations are most likely the consequence of run-away phenomena and forward scattering collisions, due to characteristic short lengths and high intensity fields present in microplasmas. Nevertheless the chemistry of the plasma environment is mostly determined in the lower energy range where reaction cross sections tend to be much larger so that energetic electrons can be viewed as loss processes which mainly affect the electron density rather than the temperature of slower electrons. At atmospheric pressure, electrons tend to assume a Maxwellian distribution and this has been observed even for microplasmas. ${ }^{49}$ Therefore the use of a Maxwellian distribution can still be justified to understand and/or satisfactorily model microplasma behavior. In our case an effective temperature is considered and is determined from spectral emission data.

In order to determine an effective electron temperature (from now on simply referred as the electron temperature or $T_{e}$ ), a very simple collisional-radiative model for argon excited levels is used. A more detailed discussion of the model and the derivation of the temperature is discussed elsewhere. ${ }^{50}$ The model assumes that population of four of the $4 p$ levels of argon is dominated by electron excitation kinetics, and spontaneous radiative decay is assumed to be the sole cause for the deexcitation from such excited energy levels. Here the $2 p_{2}, 2 p_{3}, 2 p_{6}$, and $2 p_{10}$ levels (in Paschen notation) have been chosen. It is important to underline the fact that the model is self-consistent and the description of the population and depopulation processes of only the four selected $4 p$ levels is required.

Here, it is also necessary to clarify why other processes can be neglected. It is common understanding that with increasing pressure, the contribution from neutrals in the excitation to $4 p$ levels increases. Nevertheless this is generally true only if the difference between electron temperature and gas temperature is drastically reduced. In nonequilibrium plasmas, where the electron temperature is still considerably higher than the gas temperature, the contribution of neutrals can still be neglected. ${ }^{51}$ Furthermore it is now accepted that atmospheric microplasmas maintain the nonequilibrium characteristic. ${ }^{27,28}$ All this supports the fact that electron excitation can still be dominant for an atmospheric microplasma.

The cascading contribution from higher-energy levels is also quite low as electron energy distribution generally decreases with increasing energy and this is confirmed by low emission lines from the higher $3 d$ and $5 s$ levels. In our observations, among all levels above $4 p$, only emission originating from the $5 p$ has been measured, but from these levels the only possible transitions are to populate the $4 s$ levels.
And with no surprise the $5 p$ levels are subject of excitation from metastable which justifies the high population despite a higher energy if compared with $3 d$ and $5 s$.

Other processes such as three-body recombination, radiative recombination, and stepwise excitation (excluding excitation from metastable) can all be assumed to be of minor importance due to the combined effect of a low electron density, a low ion density, and a short lifetime for the $4 p$ excited levels $(22-39 \mathrm{~ns}){ }^{52}$

Finally, the effect of radiation trapping should be commented as in atmospheric pressure could influence the recorded emission. However, it should be reminded that in this study the microplasma has a very small radius, less than $0.1 \mathrm{~mm}$, which can be considered relatively small allowing for radiation to escape the plasma volume. Moreover, radiation trapping can occur for emission from levels higher than the $4 p$ or for radiation from $4 p$ levels to metastable levels. In the latter case reabsorption is very weak and therefore radiation trapping can be considered negligible. ${ }^{45}$ In the first case, due to the self-consistency of the set of the model equations (see below) and the fact that radiation from higher levels to $4 p$ levels was not observed, optical thinness can be assumed all together.

In this scenario the balance mass conservation equation for each of the four energy levels can be written in the following simple way:

$$
n_{e} n_{g} k_{g, 2 p x}+\sum_{i=3,5} n_{e} n_{1 s i} k_{1 s i, 2 p x}=\sum_{i=5}^{2} n_{2 p x} A_{2 p x, 1 s i},
$$

and rewriting Eq. (1) for each of the $4 p$ levels considered and rearranging for $n_{e}$ we obtain

$$
\begin{gathered}
n_{e}=\frac{\sum_{i=5}^{2} n_{2 p 2} A_{2 p 2,1 s i}}{n_{g} k_{g, 2 p 2}+\sum_{i=3,5} n_{1 s i} k_{1 s i, 2 p 2}}, \\
n_{e}=\frac{\sum_{i=5}^{2} n_{2 p 3} A_{2 p 3,1 s i}}{n_{g} k_{g, 2 p 3}+\sum_{i=3,5} n_{1 s i} k_{1 s i, 2 p 3}}, \\
n_{e}=\frac{\sum_{i=5}^{2} n_{2 p 6} A_{2 p 6,1 s i}}{n_{g} k_{g, 2 p 6}+\sum_{i=3,5} n_{1 s i} k_{1 s i, 2 p 6}}, \\
n_{e}=\frac{\sum_{i=5}^{2} n_{2 p 10} A_{2 p 10,1 s i}}{n_{g} k_{g, 2 p 10}+\sum_{i=3,5} n_{1 s i} k_{1 s i, 2 p 10}},
\end{gathered}
$$

where $n$ indicates the number density, $k_{x, y}$ is the rate coefficient for the excitation from level $x$ to level $y$, and $A_{y, x}$ is the transition probability from level $y$ to level $x$. The subscript $g$ stands for ground level, $e$ for electrons, and $1 s_{x}$ and $2 p_{x}$ are 
the Paschen notations for each $3 p$ and $4 p$ energy level, respectively. The first and second terms in Eq. (1) represent, respectively, excitation by electron collision from the ground level and from the two metastable levels $\left(1 s_{3}\right.$ and $1 s_{5}$ in Paschen notation). Radiative decay from the $4 p$ levels is only allowed to the four $4 s$ levels, i.e., the two resonant levels $\left(1 s_{2}\right.$ and $1 s_{4}$ in Paschen notation) and the two metastable levels ( $1 s_{3}$ and $1 s_{5}$ in Paschen notation), which is represented by the sum on the right hand side.

Equation (2), coupled two by two, provides a system of two equations and therefore can be solved for the two metastable densities $n_{1 s 3}$ and $n_{1 s 5}$. The expressions for $n_{1 s 3}$ and $n_{1 s 5}$ found in this way can then be replaced in the same equations. At this point, in Eq. (2), $n_{g}$ and $A_{y, x}$ are known terms, where the first can be deduced from the pressure. The excited state density $n_{2 p x}$ is then determined from experimental emission intensities according to

$$
n_{2 p x}=C \frac{I_{x, y} \lambda_{x, y}}{A_{x, y}},
$$

where $C$ represents a proportionality constant as the spectrometer has been calibrated only to provide correct relative intensities; $I_{x, y}$ is the measured relative intensity, and $\lambda_{x, y}$ the corresponding wavelength.

Finally, the rate coefficients found in Eq. (2) can be calculated numerically according to

$$
\begin{aligned}
k\left(T_{e}\right) & =\int_{0}^{\infty} \sigma(\varepsilon) \sqrt{\frac{2 \varepsilon}{m}} f_{M}\left(\varepsilon, T_{e}\right) d \varepsilon \\
& \approx \sum_{i} \sigma\left(\varepsilon_{i}\right) \sqrt{\frac{2 \varepsilon_{i}}{m_{e}}} f_{M}\left(\varepsilon_{i}, T_{e}\right)\left(\varepsilon_{i}-\varepsilon_{i-1}\right),
\end{aligned}
$$

where $\sigma$ is the corresponding excitation cross section, $\varepsilon$ is the energy, $m_{e}$ is the electron mass, $f_{M}$ is the Maxwellian probability function (or the normalized energy distribution), and the term $\varepsilon_{i}$ represents the discretized values for the energy. By assuming a Maxwellian distribution, the only unknown parameter in Eq. (4) and consequently in Eq. (2) remains the effective electron temperature $T_{e}$, so that this can be used as a fitting parameter to produce the same electron density value of $n_{e}$ in all four equations of Eq. (2). In practice only two equations need to be fitted as Eq. (2) was already coupled two by two to determine metastable densities. Also, due to the constant $C$ in Eq. (3), the electron density $n_{e}$ to be matched represents a relative electron density $\left(n_{e} / C\right)$ and not an absolute value. This procedure has therefore been applied to determine $T_{e}$ at different conditions of the microplasma settings and results are discussed in the following sections.

With the same calculations just described, metastable state density of argon is also obtained; nevertheless these will not be discussed within this report. As a general observation, metastable density of each level has varied in the range between $10^{18}$ and $10^{21} \mathrm{~m}^{-3}$ and has increased when electron temperature was increasing and vice versa. The range of metastable density is in agreement with generally observed densities in ionized gas applications (around $10^{-5}$ of the ground state and $\left.10^{18} \mathrm{~m}^{-3}\right) .^{53,54}$
TABLE I. Selected $4 p$ argon levels and wavelength originating from corresponding levels.

\begin{tabular}{ccc}
\hline \hline $\begin{array}{c}\text { Level } \\
\text { (Paschen notation) }\end{array}$ & Wavelength $(\mathrm{nm})$ & Transition probabilities $\left(\mathrm{s}^{-1}\right)$ \\
\hline $2 p_{2}$ & 696.5430 & $6.39 \times 10^{6}$ \\
& 727.2935 & $1.83 \times 10^{6}$ \\
& 826.4521 & $1.53 \times 10^{7}$ \\
$2 p_{3}$ & 706.7217 & $3.80 \times 10^{6}$ \\
& 738.3980 & $8.47 \times 10^{6}$ \\
$2 p_{6}$ & 763.5105 & $2.45 \times 10^{7}$ \\
& 922.4498 & $5.03 \times 10^{6}$ \\
$2 p_{10}$ & 912.2967 & $1.89 \times 10^{7}$ \\
& 965.7786 & $5.43 \times 10^{6}$ \\
\hline
\end{tabular}

Few last comments should be added with regard to the calculation of the number densities in Eq. (3) and the rate coefficient in Eq. (4). For each one of the four $4 p$ levels that have been selected $\left(2 p_{2}, 2 p_{3}, 2 p_{6}\right.$, and $2 p_{10}$ in Paschen notation) more than one radiative path is possible with corresponding emission line. Table I shows the emission lines used for the calculations and transition probabilities used in Eq. (3) $;^{55}$ for each energy level listed the number density of Eq. (3) has been calculated using separately each one of the available emission lines and then averaged. The corresponding standard deviation of these measurements was generally around or below $10 \%$ of the average value, although in few cases reached $19 \%$.

Concerning the calculation of the rate coefficient in Eq. (4), cross section data for electron collision excitation from ground state are readily available and numerous. ${ }^{52,56-59} \mathrm{Nev}-$ ertheless there are few data relative to excitation from the metastable levels. ${ }^{53,58,60,61}$ In our calculation, to keep consistency of the cross section data, the theoretical cross sections reported from the same group have been used. ${ }^{60}$

\section{RESULTS AND DISCUSSION}

\section{A. Introduction to experimental results}

Gas temperature and electron temperature have been determined for a range of different settings to evaluate the effect of power, oxygen content, and flow rate on the two temperature parameters. All results reported here are average values of at least nine different measurements. Standard deviations varied from $12 \%$ down to $2 \%$ of the average value. It has been noticed that measurements presented a small drift, which has been the main cause of the higher standard deviations. This can be probably be ascribed to wire surface degradation. Nevertheless the trend of all measurement has been preserved for all conditions.

\section{B. Effect of flow rate}

Flow rate was varied between 10 and 20 SCCM with no appreciable difference in both gas temperature and electron temperature (therefore not shown here). Likely, the relatively low flow rate variation neither introduced noticeable differences in the pressure within the quartz vessel nor contributed 


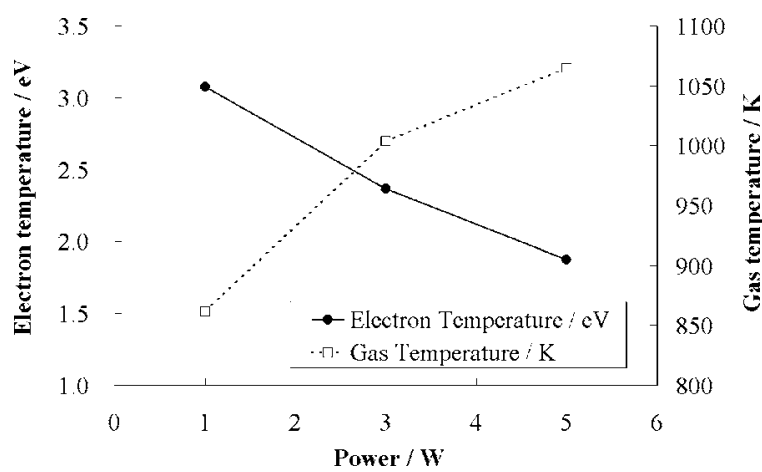

FIG. 6. Gas temperature and electron temperature for argon microplasma at 20 SCCM flow rate.

effectively to change the rate of particle loss processes. In the future, more investigations will be performed at higher flow rates as they become interesting for the specific material processing application.

\section{Effect of power}

The effect of power is shown in Fig. 6 for a pure argon microplasma. The sustaining UHF power was set at 1,3 , and $5 \mathrm{~W}$ while flow rate was constant at 20 SCCM. The effect of higher power on gas temperature may seem quite obvious, as the input of more power would correspond to more energy in the plasma system. Nevertheless the reading of the electron temperature makes the overall interpretation less immediate. It has to be noticed that when the power was increased, the plasma envelope around the titanium wire was also increased, i.e., increasing the power the plasma was covering more and more of the wire length (see Fig. 4). So that the overall surface area available for electron emission from the wire was increased and also field lines were increased in length.

The extension of the plasma can be justified by a stronger electric field being able to break down the gas in more remote wire positions farther from the copper tube. Once the plasma is formed, the electric field is redistributed consistently with the distribution of the charges. In this process several phenomena should be considered. The field in the plasma volume with exclusion of boundary zones is probably weakened due to longer field path and higher electron density; as a consequence energy exchanged between the field and the charged particles is also weakened. A decreasing electron temperature, as observed in Fig. 6, is therefore justified and would not drastically affect the ionization efficiency by electron collisions at high electron energies. It follows that overall, electron temperature is reduced but the ionization rate is increased by a larger source of electrons at the wire surface, which then leads to an increased ion density.

Ultimately, energy transfer from ions to neutral argon atoms is the controlling factor for the gas temperature, as electrons are incapable of transferring momentum to the much heavier neutral atoms. Obviously diffusion and heat exchange with surrounding walls play a major role, but for this analysis these conditions are unchanged. Argon atoms in

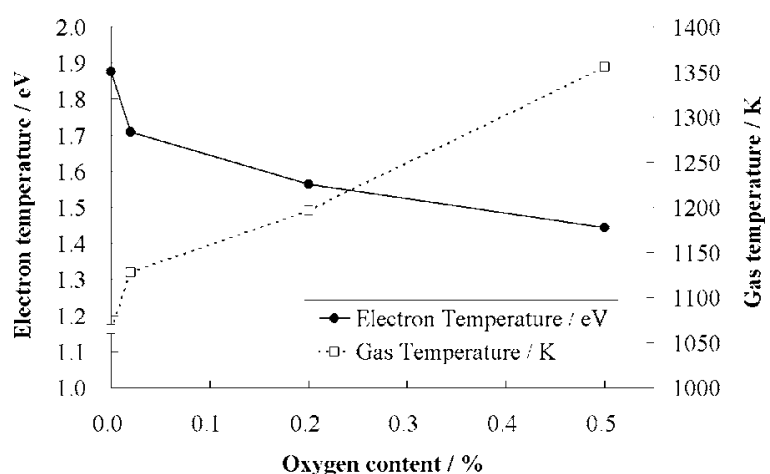

FIG. 7. Gas temperature and electron temperature for microplasma sustained with $5 \mathrm{~W}$ at 20 SCCM flowing argon-oxygen mixtures.

metastable states could also contribute to gas heating; nevertheless these are much more sensitive to a decreasing electron temperature.

In summary, as the UHF power is increased, electron temperature is reduced, but the ionization rate is enhanced by a larger fraction of electrons supplied by a larger surface area at the wire. An increased density of argon ions then increases the gas temperature. It is therefore shown that increasing power does not always lead to an enhanced electron activity to produce a more reactive environment. In this specific case the microplasma volume and surface contact walls have played an important role in channeling energy into an overall gas heating.

\section{Effect of oxygen content}

Here we have studied the microplasma sustained by $5 \mathrm{~W}$ UHF power in 20 SCCM flowing argon with different concentrations of added oxygen. The presence of oxygen makes all plasma processes rather complex, even in a simple binary gas mixture with inert argon. ${ }^{32,62-65} \mathrm{~A}$ full understanding requires a detailed spectral analysis with a more sensitive and better resolution spectrometer; here the aim is to highlight some characteristics and provide a qualitative evaluation.

The electron temperature is observed to decrease sharply with little $\mathrm{O}_{2}$ added (Fig. 7). Introduction of oxygen in the gas mixture certainly contributes to energy losses for electrons; the number of excitation, ionization, and dissociation processes is quite large and many of the processes that involve atomic and/or molecular oxygen require relatively low activation energies ( 4.5 up to $14.7 \mathrm{eV}) .^{62}$ These all contribute to lower the electron temperature with just little oxygen introduced.

Due to the electron-induced processes with oxygen, less electron energy is available for ionization and generation of argon ions. It follows that argon ions are likely to have reduced densities when oxygen is introduced and cannot be considered responsible for an increase in gas temperature in this case. Nevertheless, electron collisions with oxygen efficiently promote a variety of positive and negative ions $\left(\mathrm{O}^{+}\right.$, $\left.\mathrm{O}_{2}^{+}, \mathrm{O}_{4}^{+}, \mathrm{O}_{3}^{-}, \mathrm{O}_{2}^{-}, \mathrm{O}^{-}\right){ }^{62}$ These oxygen-related ions can all effectively contribute to increasing gas temperature. In particular, dissociative electron attachment leading to $\mathrm{O}^{-}+\mathrm{O}$ is very effective and is activated at energy as low as $5 \mathrm{eV}^{66}$ this dissociative process can reduce electron energy, decrease 
electron density, and contribute to channel energy into heating the gas all at the same time. Also, some exothermic oxidation processes with titanium atoms may have contributed to increasing gas temperature.

The characteristic properties of oxygen and its electronegativity seem to be responsible for transferring energy from electrons to an overall increased gas temperature.

\section{CONCLUSIONS}

A low-power microplasma configuration is presented and its appearance is analyzed. The ignition and maintenance of the microplasma are not fully understood in all its contributing factors and will be the subject of future work. Nevertheless, considering the ultrahigh frequency employed and the observed behavior, this microplasma does present interesting aspects. Also, it is shown that the microplasma is in nonequilibrium and it can be easily sustained in atmospheric plasma glow discharge (APGD) mode with different gas mixtures of argon and oxygen.

Gas temperature has been determined with an established spectroscopy technique and compared with the electron temperature calculated using an analytical solution of a simple collisional-radiative model. The model requires few assumptions such as a Maxwell distribution of energies for electrons but it allows easy computation of the electron temperature and argon metastable densities. Some future work is also directed in providing a technique to determine the true electron energy distribution. The results have confirmed that at atmospheric pressure this microplasma is in nonequilibrium.

Gas temperature and electron temperature changes have been analyzed when power, flow, and oxygen content have been varied. The latter parameter, i.e., oxygen concentration, is of particular importance for many applications. Application of this atmospheric microplasma to material processing depends on the ability to control gas temperature providing a desired processing environment and at the same time, control over the electron energy is necessary to supply radicals. Results have revealed that on one side gas temperature is easily increased. On the other side tailoring of a reactive environment requires expert control and monitoring of a combination of factors (e.g., gas and electron temperatures, electron density, gas composition, etc.).

This work has highlighted the close relation of the plasma parameters and that a combined set of diagnostics can be extremely valuable in understanding plasma processes. Emission spectroscopy contains a large amount of information and combined with simple computational techniques; multiple diagnostics are possible with the same set of experimental data. Further development of spectral analysis can become extremely useful for microplasmas, where diagnostic techniques and relative results are still limited.

\section{ACKNOWLEDGMENTS}

This research was supported by JSPS fellowship and Grant-in-Aid for scientific research from the Japanese Ministry of Education, Culture, Sports, Science and Technology.
We would also like to thank Professor Bartschatt for providing us with the numerical results of the theoretical cross section calculations. ${ }^{60}$

${ }^{1}$ J. P. Boeuf, C. Punset, A. Hirech, and H. Doyeux J. Phys. IV 7, 3 (1997). ${ }^{2}$ J. P. Boeuf, J. Phys. D 36, R53 (2003).

${ }^{3}$ K. H. Becker, K. H. Schoenbach, and J. G. Eden, J. Phys. D 39, R55 (2006).

${ }^{4}$ C. Tendero, C. Tixier, P. Tristant, J. Desmaison, and P. Leprince, Spectrochim. Acta, Part B 61, 2 (2006).

${ }^{5}$ R. M. Sankaran and K. P. Giapis, Appl. Phys. Lett. 79, 593 (2001).

${ }^{6}$ T. Ito and K. Terashima, Thin Solid Films 390, 234 (2001).

${ }^{7}$ T. Ito, T. Izaki, and K. Terashima, Thin Solid Films 386, 300 (2001).

${ }^{8}$ R. M. Sankaran and K. P. Giapis, J. Appl. Phys. 92, 2406 (2002).

${ }^{9}$ Y. Shimizu, T. Sasaki, T. Ito, K. Terashima, and N. Koshizaki, J. Phys. D 36, 2940 (2003)

${ }^{10}$ T. Kikuchi, Y. Hasegawa and H. Shirai, J. Phys. D 37, 1537 (2004).

${ }^{11}$ T. Ichiki, R. Taura, and Y. Horiike, J. Appl. Phys. 95, 35 (2004).

${ }^{12}$ Y. Shimizu, T. Sasaki, C. Liang, A. C. Bose, T. Ito, K. Terashima, and N. Koshizaki, Chem. Vap. Deposition 11, 244 (2005).

${ }^{13}$ Y. Shimizu, T. Sasaki, A. C. Bose, K. Terashima, and N. Koshizaki, Surf. Coat. Technol. 200, 4251 (2006).

${ }^{14}$ H. Shirai, T. Kobayashi, and Y. Hasegawa, Appl. Phys. Lett. 87, 143112 (2005).

${ }^{15}$ Y. Shimizu, A. C. Bose, T. Sasaki, D. Mariotti, K. Kirihara, T. Kodaira, K. Terashima, and N. Koshizaki, Trans. Mater. Res. Soc. Jpn. 31, 463 (2006).

${ }^{16}$ Y.-M. Sung and T. Sakoda, Surf. Coat. Technol. 197, 148 (2005).

${ }^{17}$ A. Rahman, A. P. Yalin, V. Surla, O. Stan, K. Hoshimiya, Z. Yu, E. Littlefield, and G. J. Collins, Plasma Sources Sci. Technol. 13, 537 (2004).

${ }^{18}$ Y. Takao and K. A. Ono, Plasma Sources Sci. Technol. 15, 211 (2006).

${ }^{19}$ A. M. Bilgic, E. Voges, U. Engel, and A. C. Broekaert, J. Anal. At. Spectrom. 15, 579 (2000)

${ }^{20}$ J. Franzke, K. Kunze, M. Miclea, and K. Niemax, J. Anal. At. Spectrom. 18, 802 (2003)

${ }^{21}$ V. Karanassios, Spectrochim. Acta, Part B 59, 909 (2004).

${ }^{22}$ M. Miclea, K. Kunze, J. Franzke, and K. Niemax, Spectrochim. Acta, Part B 57, 1585 (2002).

${ }^{23}$ C. Brede, S. Pedersen-Bjergaard, E. Lundanes, and T. Greibrokk, J. Anal. At. Spectrom. 15, 55 (2000).

${ }^{24}$ J. C. T. Eijkel, H. Stoeri, and A. Manz, Anal. Chem. 71, 2600 (1999).

${ }^{25}$ J. G. Eden, S.-J. Park, N. P. Ostrom, and K.-F. Chen, J. Phys. D 38, 1644 (2005).

${ }^{26}$ J. G. Eden and S.-J. Park, Plasma Phys. Controlled Fusion 47, B83 (2005).

${ }^{27}$ I. E. Kieft, E. P. van der Laan, and E. Stoffels, New J. Phys. 6, 149 (2004).

${ }^{28}$ R. Rahul et al., J. Phys. D 38, 1750 (2005).

${ }^{29}$ J. J. Shi and M. G. Kong, Phys. Rev. Lett. 96, 105009 (2006).

${ }^{30}$ H. Akatsuka, Sci. Technol. Adv. Mater. 5, 651 (2004).

${ }^{31}$ A. Al-Jalai and M. A. Khan, J. Appl. Phys. 99, 033302 (2006).

${ }^{32}$ S. H. Lee, F. Iza, and J. K. Lee, Phys. Plasmas 13, 057102 (2006).

${ }^{33}$ D. Robinson, J. Quant. Spectrosc. Radiat. Transf. 4, 335 (1964).

${ }^{34}$ D. M. Phillips, J. Phys. D 8, 507 (1975).

${ }^{35}$ S. Pellerin, J. M. Cormier, F. Richard, K. Musiol, and J. Chapelle, J. Phys. D 29, 726 (1996).

${ }^{36}$ C. de Izarra, J. Phys. D 33, 1697 (2000).

${ }^{37}$ F. Iza and J. A. Hopwood, IEEE Trans. Plasma Sci. 32, 498 (2004).

${ }^{38}$ H. Rabat and C. de Izarra, J. Phys. D 37, 2371 (2004).

${ }^{39}$ R. Ono, M. Nifuku, S. Fujiwara, S. Horiguchi, and T. Oda, J. Appl. Phys. 97, 123307 (2005).

${ }^{40}$ Z. H. Wang, P. D. Swift, A. J. Studer, D. R. McKenzie, B. W. James, and I. S. Falconer, Appl. Opt. 29, 5145 (1990).

${ }^{41}$ C. Nouvellon, S. Konstantinidis, J. P. Dauchot, M. Wautelet, P. Y. Jouan, A. Ricard, and M. Hecq, J. Appl. Phys. 92, 32 (2002).

${ }^{42}$ A. Belkind, W. Zhu, J. Lopez, and K. Becker, Plasma Sources Sci. Technol. 15, S17 (2006).

${ }^{43}$ G. M. Petrov, J. L. Giuliani, A. Dasgupta, K. Bartschat, and R. E. Pechacek, J. Appl. Phys. 95, 5248 (2004).

${ }^{44}$ C. Lao, J. Cotrino, A. Palmero, A. Gamero, and A. R. Gonzalez-Elipe, Eur. Phys. J. D 14, 361 (2001).

${ }^{45}$ M. J. Schabel, V. M. Donnelly, A. Kornblit, and W. W. Tai, J. Vac. Sci. Technol. A 20, 555 (2002).

${ }^{46}$ D. Mariotti, P. Maguire, C. M. O. Mahony, and J. McLaughlin, Plasma Sources Sci. Technol. 13, 576 (2004) 
${ }^{47}$ U. Fantz et al., Nucl. Fusion 46, S297 (2006).

${ }^{48}$ G. C. Wilson and Y. B. Gianchandani, J. Appl. Phys. 94, 2845 (2003).

${ }^{49}$ A. Kono and K. Iwamoto, Jpn. J. Appl. Phys., Part 2 43, L1010 (2004).

${ }^{50}$ D. Mariotti, Y. Shimizu, T. Sasaki, and N. Koshizaki, Appl. Phys. Lett. 89, 201502 (2006).

${ }^{51}$ J. Bacri and A. M. Gomes, J. Phys. D 11, 2185 (1978)

${ }^{52}$ J. B. Boffard, C. C. Lin, and C. A. DeJoseph, Jr, J. Phys. D 37, R143 (2004).

${ }^{53}$ G. A. Piech, J. B. Boffard, M. F. Gehrke, L. W. Anderson, and C. C. Lin, Phys. Rev. Lett. 81, 309 (1998).

${ }^{54}$ T. Ito, H. Nishiyama, K. Terashima, K. Sugimoto, H. Yoshikawa, H. Takahashi, and T. Sakurai, J. Phys. D 37, 445 (2004).

${ }^{55} \mathrm{http}: / /$ physics.nist.gov/PhysRefData/ASD/index.html

${ }^{56}$ J. K. Ballou and C. C. Lin, Phys. Rev. A 8, 1797 (1973).

${ }^{57}$ J. E. Chilton, J. B. Boffard, R. S. Schappe, and C. C. Lin, Phys. Rev. A 57, 267 (1998).
${ }^{58}$ A. Dasgupta, M. Blaha, and J. L. Giuliani, Phys. Rev. A 61, 012703 (1999).

${ }^{59}$ A. Yanguas-Gil, J. Cotrino, and L. L. Alves, J. Phys. D 38, 1588 (2005). ${ }^{60} \mathrm{~K}$. Bartschat and V. Zeman, Phys. Rev. A 59, R2552 (1999).

${ }^{61}$ J. B. Boffard, G. A. Piech, M. F. Gehrke, L. W. Anderson, and C. C. Lin, Phys. Rev. A 59, 2749 (1999).

${ }^{62}$ M. A. Lieberman and A. J. Lichtenberg, Principles of Plasma Discharges and Material Processing (Wiley, Hoboken, NJ, 2005), pp. 270-271.

${ }^{63}$ C. Soria, F. Pontiga, and A. Castellanos, Plasma Sources Sci. Technol. 13, 95 (2004).

${ }^{64}$ J. T. Gudmundsson, J. Phys. D 37, 2073 (2004).

${ }^{65}$ M.-H. Lee, S.-H. Jang, and C.-W. Chung, Phys. Plasmas 13, 053502 (2006).

${ }^{66}$ K. H. Becker, U. Kogelschatz, K. H. Schoenbach, and R. J. Barker, Nonequilibrium Air Plasmas at Atmospheric Pressure (IOP, Bristol, 2005), p. 129. 